\title{
Primary ciliary dyskinesia
}

The integrity of healthy respiratory epithelium is maintained, at least in part, by trapping inhaled particles and microorganisms in a protective blanket of mucus which is transported towards the oropharynx by the coordinated action of the underlying ciliated cells. Ciliary dyskinesia is a recently recognised condition in which there is profound disturbance of this clearance mechanism because of the ineffective beating of cilia, and results in the stasis of airway mucus. The incidence of this disorder has been estimated at between 1 in 15000 and 30000 and it is thought to be inherited as an autosomal recessive. It was first described in three males with Kartagener's syndrome (situs inversus, bronchiectasis, and sinusitis) who were found to have immotile spermatozoa as well as recurrent respiratory infections. ' It was suggested that as sperm tails and respiratory cilia have similar ultrastructure there might be a generalised defect causing immotility of both sperm tails and cilia. The finding of impaired tracheobronchial clearance, a shared abnormality of ciliary and sperm tail ultrastructure, and the observation of absent ciliary motility in bronchial biopsy specimens strongly supported this hypothesis. Cilia and sperm tails both contain nine pairs of protein filaments arranged around a central pair. Attached to each of the nine outer doublets are structures containing adenosine triphosphatase called dynein arms which are thought to be responsible for normal ciliary beating by sliding on the adjoining inner microtubule of the next pair. It was these dynein arms that were noted to be missing from the cilia and sperm tails of patients with Kartagener's syndrome. The original term describing this condition-immotile cilia syndrome-is, in fact, a misnomer. A limited amount of ciliary activity may occur in patients with Kartagener's syndrome because absolute deficiency of both sets of dynein arms is unusual. When it occurs, however, ciliary movement is abnormal, usually very slow and stiff, and the beating of adjacent ciliated cells is not coordinated. It has been suggested that dextrocardia occurs in this condition because the direction of rotation of the archenteron is random in the absence of ciliary beating on embryonic cells so that there will be only a $50 \%$ chance that the dextral spiral (and hence normal cardiac situs) will occur. ${ }^{1}$ The spectrum of primary ciliary dyskinesia also includes patients without dextrocardia but who have an identical structural abnormality of their cilia. Kartagener's syndrome is therefore a subgroup of primary ciliary dyskinesia-with the important implication that only some patients will be recognisable clinically and radiologically on the basis of situs inversus.

\section{Clinical features}

Although cilia line neural ependyma, cervix, uterus, fallopian tubes, and epididymis, ${ }^{2}$ it is in the respiratory tract that the consequences of their dysfunction are most obvious. The nose, paranasal sinuses, middle ear, and bronchi all rely on effective ciliary activity to remove secretions. Because primary ciliary dyskinesia is congenital, symptoms may be present from birth, and indeed the disorder may present in the neonatal period with acute respiratory distress. Chronic cough and rhinorrhoea frequently occur throughout childhood and have usually been present from birth. Sinus washouts may be recommended and performed on many occasions because of symptoms from infected, radio-opaque sinuses. Fluid accumulates in the middle ear, due to eustachian tube dysfunction, and often causes a mild conductive hearing loss. If grommets are inserted they continue to discharge until they are removed or extruded, after which the fluid usually reaccumulates. Similar impairment of lower respiratory tract clearance results in persistent cough, recurrent chest infections, and eventually bronchiectasis. Normal ciliary beating in the fallopian tube does not seem to be vital to ovum transport and women with this condition are usually fertile. Most men are infertile because of the coexistent sperm tail defect, although there are well documented cases in which puzzling discordance exists between ciliary and sperm tail ultrastructure. ${ }^{3}$

\section{Ciliary ultrastructure}

Not all cilia from patients with primary ciliary dyskinesia show complete absence of dynein arms. The deficiency, which may only be partial, may affect outer or inner arms, or both. There are also families described in which the radial spokes of cilia are missing ${ }^{4}$ or one pair of microtubules is transposed to replace the central pair. ${ }^{5}$ More recently there have been reports of patients with normal ciliary ultrastructure ${ }^{6}$ and also of patients apparentiy with ciliary aplasia throughout the respiratory tract. ${ }^{78}$ Despite differing morphological abnormalities, the clinical syndromes expressed were similar. Affected family members have identical ultra- 
structural abnormalities which tends to support the concept of a genetically determined heterogeneity of defects all leading to impaired ciliary movement and mucus clearance.

\section{Diagnosis}

Ideally, a suspected diagnosis of ciliary dyskinesia should be confirmed by the finding of absent or severely impaired mucociliary clearance and of functional and morphological abnormalities of respiratory cilia. Mucociliary clearance measurement by inhalation of radiolabelled particles is not widely available and requires a controlled inspiratory manoeuvre for adequate bronchial deposition-which is not generally feasible for those under the age of 10 years. Similarly, nasal mucociliary clearance using a saccharin test is not reliable below this age. For some years the mainstay of diagnosis was the finding of ultrastructural abnormalities in tissue obtained by biopsy of the inferior turbinate using local anaesthesia. Although this approach provides adequate samples for electron microscopy, the procedure is traumatic, invasive, and may be complicated by haemorrhage. Furthermore, the anaesthetic may inhibit ciliary beating making light microscopic observation of function difficult to interpret. The finding of normal ciliary ultrastructure will not definitively exclude a diagnosis of ciliary dyskinesia so the presence or absence of ciliary beating must be observed. Our current practice is to obtain cilia from the inferior turbinate without anaesthesia using a cytology brush, suspend the cilia in tissue culture medium, and examine them by light microscopy in a sealed glass preparation at $37^{\circ} \mathrm{C}$. This usually yields many ciliated cells and allows assessment of beat pattern, degree of immotility, ${ }^{6}$ and measurement of ciliary beat frequency using a photometric technique. ${ }^{9}$ Nasal cilia are routinely used for diagnosis because of their accessibility, but the defect in this condition is generalised throughout the respiratory tract even if symptoms predominate at one or other end of it. In some cases of primary ciliary dyskinesia, many of the cilia may be beating, but the pattern of this beating is abnormal and uncoordinated and metachronal waves are not seen.

\section{Treatment}

Unfortunately there is little in the way of specific treatment to be offered. The standard advice given to parents of children with bronchiectasis-prompt treatment of infection, adequate bronchodilator treatment, and regular daily postural drainage for life-are particularly appropriate to this group of patients. With no effective mucociliary clearance of their own, they seem to rely entirely on the effects of gravity and cough to keep their airways free of mucus. Because bronchiectasis seems to progress with repeated infections, these must be recognised promptly and treated effectively, checking with vigilant follow up. Mucolytic agents (for example alkaline nasal douches) and anti-inflammatory agents may be effective for nasal symptoms, and fortunately hearing loss is usually mild and does not require specific treatment. Surgery, to reduce the size of the inferior turbinates, may improve nasal obstruction considerably and inferior nasal antrostomies will aid gravity drainage of maxillary antra. Arguably, however, the main importance of the nasal symptoms is that they should alert the clinician to this condition early in life so that prophylactic measures may be instituted before irreversible lung damage has occurred. It seems likely, though unproved, that such treatment will delay if not prevent the onset of bronchiectasis. A child with severe bronchiectatic symptoms because of a chronically collapsed, infected lobe may benefit from a localised pulmonary resection as otherwise such a lobe may act as a 'sump' from which infected material can be spread throughout the bronchial tree. This approach is only feasible if bronchography confirms localised disease.

Lung function, although impaired, remained surprisingly stable in one longitudinal study of children with this condition, ${ }^{10}$ suggesting that cough and perhaps as yet unrecognised clearance mechanisms in the lung may be of importance in allowing most of these children to lead relatively healthy lives of near normal length.

\footnotetext{
References

1 Afzelius BA. A human syndrome caused by immotile cilia. Science 1976;193:317-9.

2 Afzelius BA. The immotile cilia syndrome and other ciliary diseases. Int Rev Exp Pathol 1979;19:1-43.

3 Jonsson MS, McCormick JR, Gillies CG, Gondos B. Kartagener's syndrome with motile spermatozoa. $N$ Engl $J$ Med 1982;307:1131-3

${ }^{4}$ Sturgess JM, Chao J, Wong J, Aspin N, Turner JAP. Cilia with defective radial spokes. A cause of human respiratory disease. N Engl J Med 1979;300:53-6.

5 Sturgess JM, Chao J, Turner JAP. Transposition of ciliary microtubules. Another cause of impaired ciliary motility. $N$ Engl J Med 1980;303:318-22.

6 Greenstone M, Dewar A, Cole PJ. Ciliary dyskinesia with normal ultrastructure. Thorax 1983;38:875-6.

${ }^{7}$ Götz M, Stockinger L. Aplasia of respiratory tract cilia. Lance $1983 ; \mathbf{i}: 1283$

${ }^{8}$ Fonzi L, Lungarella G, Palatresi R. Lack of kinocilia in the nasal mucosa in the immotile-cilia syndrome. Eur J Respir Dis 1982;63:558-63.
} 
${ }^{9}$ Rutland J, Cole PJ. Non-invasive sampling of nasal cilia for measurement of beat frequency and study of ultrastructure. Lancet 1980;ii:564-5.

10 Corkey CWB, Levison H, Turner JAP. The immotile cilia syndrome-a longitudinal survey. Am Rev Respir Dis 1981;124:544-8.

M Greenstone ANd P J Cole Cardiothoracic Unit, Brompton Hospital, Fulham Road, London SW3 6HP

\section{Fifty years ago}

\section{Infant welfare centres}

'DR Reginald Miller (London), in opening a discussion on the use and abuse of infant welfare centres, said that the latest figures available to indicate the size of the movement related to the end of 1932 . There were then 2783 centres in England, including 218 in London. Of these 749 were under voluntary bodies and the rest under local authorities. Compared with the previous year the total number had increased by 32 , the voluntary centres had diminished by 88 and the municipal increased by 120 . In 1932 the number of children under one year attending for the first time was 318166 , amounting to 57.8 per cent of the notified births. He argued that the movement now showed all the hall-marks of bureaucratic control, and that the right people to be in charge were those experienced in paediatrics and not those specially instructed in public health. His own view of the movement could be summed up in Whistler's dictum on Wilde: "He has no enemies, but his friends dislike him very much".'

(How right he was. How right he is today. RONALD ILLINGWORTH)

Proceedings of the Seventh Annual Meeting of the British Paediatric Association. Archives of Disease in Childhood 1934;9:198. 